\title{
Manejo del Triage Obstétrico y Código Mater en el Estado de México
}

\section{Obstetric Triage and Management Mater Code in the State of Mexico}

LEO Rosa María Alvarado Berrueta,•

LEO Margarita Arroyo Vázquez,•

LE Celeste Hernández Pérez,•

LEO Marina Vélez Calderón•

EEP Patricia Márquez Torres.•

\section{Resumen}

A pesar de las varias iniciativas de solución que han sido promovidas en años recientes, la muerte de mujeres durante el embarazo, el alumbramiento, y el postparto sigue siendo un problema grave. El número de muertes maternas ha estado disminuyendo desde el año 2000, sin embargo la tasa aún se encuentra lejos del objetivo de las Metas del Milenio. De acuerdo a la OMS, prevalecen las tres principales causas de mortalidad materna: trastornos hipertensivos del embarazo, las hemorragias durante el evento obstétrico y sepsis y otras infecciones puerperales.

El ISEM consciente de la gran responsabilidad que esta población demanda, implementó una serie de estrategias y protocolos para proveer a las mujeres embarazadas con atención oportuna en la detección de factores de riesgo que determinen un diagnóstico y tratamiento temprano para contribuir a reducir la mortalidad materna. Los protocolos implementados son dos: Triage Obstétrico y Código Mater. El Objetivo de este artículo es difundir los protocolos y estrategias ya establecidos, que han permitido agilizar la atención en la gestante y disminuir la mortalidad materna en el Hospital General de Ecatepec "Las Américas" en el estado de México. Conclusiones: La evidencia sugiere que los protocolos y estrategias implementados contribuyeron significativamente a reducir las muertes maternas en el Hospital. Este resultado se basa en la continúa capacitación del equipo multidisciplinario de salud para responder a las emergencias obstétricas.

-Estudiantes de posgrado de Enfermería Perinatal ..Coordinadora de posgrado de Enfermería Perinatal

FeCHA DE RECIBIDO: 7 ENERO 2012

FeCha DE ENVIAdo: 23 DE FEBRERo 2012

Fecha de ACEPTAdo: 4 DE MARZO 2012 


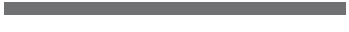 \\ Key \\ Words: \\ Maternal mortality, \\ Perinatal Care, \\ Obstetrical Nursing, \\ risk, protocol, code.}

ABStract

Despite the various initiatives for a solution which have been promoted in recent years, the death of women during pregnancy, childbirth and postpartum remains an unsolved problem. Although, the number of maternal deaths has been decreasing since the year 2000, this rate is still far from the target of the Millennium Goals. According to the WHO, the three major causes of maternal mortality are: hypertensive disorders of pregnancy, severe bleeding during the obstetric event, and sepsis.

The ISEM, aware of the great responsibility which this population demands, implemented a series of strategies and protocols in order to provide pregnant women with timely attention, detect risk factors, determine early diagnoses, and offer appropriate treatments to further contribute to reduce the maternal mortality; the protocols are: The Obstetric Triage and The Mater Code. Objective: To disseminate the established protocols and strategies those have enabled faster care in pregnant women and reduce maternal mortality in General Hospital Ecatepec "The Americas" in the state of Mexico. Conclusions: The evidence suggests that the protocols and strategies implemented significantly contributed to reduce the Hospital's maternal deaths. This outcome can also be attributed to the continued training of the multidisciplinary health team in the response of obstetric emergencies.

\section{INTRODUCCIÓN}

En la actualidad la mortalidad materna continúa siendo un problema de salud pública a nivel mundial, la muerte de las mujeres durante el embarazo, parto y puerperio está considerado un problema no resuelto $^{1}$ ello a pesar de los avances y metas que se plantearon a partir de los objetivos de desarrollo del milenio (ODM). ${ }^{2}$

En particular el quinto de estos objetivos es mejorar la salud materna y como metas a alcanzar entre los años 1990 y 2015 está reducir en tres cuartas partes la razón de mortalidad materna, lo que significa llevar el valor de este indicador a 22.6 por cien mil nacidos vivos para el año $2015 .^{3}$

A pesar que desde el año 2000 se ha observado una tendencia a la baja de este indicador, una realidad es que aun se está lejos de cumplir la meta fijada y prevalecen las tres principales causas de muerte materna dadas a conocer por la $\mathrm{OMS}^{1}$ : trastornos hipertensivos del embarazo, hemorragias durante el evento obstétrico y sepsis y otras infecciones puerperales. Tal realidad es una muestra que desafortunadamente las políticas y los programas ejecutados hasta la fecha no han sido lo suficientemente eficaces y exitosos.

La mortalidad materna es definida por la OMS como la muerte de una mujer durante el embarazo, el parto o dentro de los 42 días después de su terminación debido a cualquier causa relacionada con el embarazo. ${ }^{1}$

Este fenómeno está íntimamente relacionado con dos aspectos importantes; el primero es la injusticia social, la inequidad de género y la pobreza; el segundo con problemas en la clasificación que se da a la urgencia obstétrica por el personal de salud a cargo. ${ }^{4}$

En nuestro país, en 2009 de acuerdo al número absoluto de defunciones, son nueve las entidades federativas (Estado de México, Jalisco, Veracruz, Puebla, Chiapas, Distrito Federal, Guerrero, Oaxaca y Guanajuato) donde ocurrió el $62 \%$ de la mortalidad materna ${ }^{5}$ (Tabla 1). Comparativamente el Estado de México presentó el mayor número de muertes maternas, lo que representa casi el doble que Veracruz, estado que le sigue en orden de frecuencia. En cambio, si se considera la Razón de Mortalidad Materna $(\mathrm{RMM}=$ número de muertes maternas por cada cien mil nacidos vivos), el Estado que tiene la más baja es Colima (29.5) junto con los Estados del norte del país, con excepción de Chihuahua, los cuales tienen una RMM que oscila entre 30 y 45 . Los estados de Guerrero, Oaxaca, Chihuahua, Morelos y Chiapas presentan la más alta RMM entre 108 y 75.

De acuerdo a este indicador el estado de México se ubica en un nivel intermedio, con una RMM de 
63.3; no obstante, atendiendo a que es el que reporta un mayor número de muertes maternas, ha merecido atención en la última década, por lo que profesionales de la salud y au- toridades del sector han planteado propuestas y emprendido acciones con el objetivo de reducir este número. ${ }^{6-8}$ Lo anterior tomando como marco las metas establecidas en los

\section{Tabla 1. Razón de Mortalidad Materna por Entidad Federativa, 2009*}

\begin{tabular}{|c|c|c|}
\hline ENTIDAD FEDERATIVA & DEFUNCIONES & $\mathrm{RMM}^{*}$ \\
\hline Estados Unidos Mexicanos & 1207 & 62.2 \\
\hline Aguascalientes & 13 & 57.7 \\
\hline Baja California & 30 & 53.8 \\
\hline Baja California Sur & 5 & 51.6 \\
\hline Campeche & 8 & 55.5 \\
\hline Coahuila & 15 & 31.9 \\
\hline Colima & 3 & 29.5 \\
\hline Chiapas & 71 & 75.0 \\
\hline Chihuahua & 55 & 92.4 \\
\hline Distrito Federal & 72 & 55.4 \\
\hline Durango & 13 & 45.0 \\
\hline Guanajuato & 60 & 60.9 \\
\hline Guerrero & 68 & 107.8 \\
\hline Hidalgo & 30 & 67.3 \\
\hline Jalisco & 81 & 63.5 \\
\hline México & 166 & 63.1 \\
\hline Michoacán & 41 & 55.9 \\
\hline Morelos & 23 & 80.7 \\
\hline Nayarit & 12 & 71.3 \\
\hline Nuevo León & 25 & 33.0 \\
\hline Oaxaca & 66 & 98.3 \\
\hline Puebla & 78 & 70.1 \\
\hline Querétaro & 12 & 37.2 \\
\hline Quintana Roo & 10 & 38.5 \\
\hline San Luis Potosí & 28 & 59.2 \\
\hline Sinaloa & 18 & 40.0 \\
\hline Sonora & 21 & 47.9 \\
\hline Tabasco & 23 & 60.1 \\
\hline Tamaulipas & 24 & 43.0 \\
\hline Tlaxcala & 11 & 50.0 \\
\hline Veracruz & 93 & 72.5 \\
\hline Yucatán & 15 & 44.9 \\
\hline Zacatecas & 17 & 66.0 \\
\hline
\end{tabular}

*Razón de Mortalidad Materna.

Fuente: Secretaría de Salud. Subsecretaría de prevención y promoción de la salud. Dirección general de epidemiología. México, D.F. Junio, $2011^{5}$

Objetivos del Milenio propuestos por la ONU, ${ }^{3}$ que para el caso del estado de México se tendría que reducir de una razón de 75.05 a una de 18.77. ${ }^{9}$

En el año 2009 en 8 municipios del Estado de México la razón de mortalidad materna fue superior a 100 , sin embargo, cuando se considera el número absoluto de muertes maternas son tres los municipios con mayor número: Ecatepec, Toluca y Nezahualcoyotl. (Tabla 2)

De acuerdo con el Observatorio de la mortalidad materna en México para el año 2010 entre las principales causas de mortalidad materna se ubicaron, en primer lugar los trastornos hipertensivos del embarazo, en segundo lugar las hemorragias obstétricas y en un tercer lugar el aborto (el cual puede desencadenar en hemorragia y/o sépsis). ${ }^{11}$

Es importante recalcar que las mujeres que fallecen en los hospitales en algunos casos es debido a fallas en la atención de la emergencia obstétrica (médica o quirúrgi$\mathrm{ca})^{7}$ por lo que durante los últimos años se han implementado estrategias que en forma consecutiva buscan disminuir la morbilidad y mortalidad materna. De éstas, el Triage Obstétrico y el Código Mater representan una experiencia factible. Para que estos planes de acción tengan un éxito mayor deben considerarse otros factores importantes como lo son: fallas en el diagnóstico, escases de personal, déficit administrativo con recursos insuficientes, falta de banco de sangre en algunas unidades médicas e incluso deficiencias o carencias en medios de transporte lo que en consecuencia provoca retraso en la atención (en este caso de la paciente obstétrica). 
Tabla 2. Defunciones maternas y Razón de mortalidad materna por municipio 2009.*

\begin{tabular}{|c|c|c|c|}
\hline Municipios & $\begin{array}{c}\text { Muertes } \\
\text { Maternas } 2009\end{array}$ & $\begin{array}{l}\text { Nacidos vivos } \\
\text { estimados } 2009 \\
\text { por CONAPO }\end{array}$ & $\begin{array}{l}\text { Razón de Mortalidad } \\
\text { Materna x 100,000 } \\
\text { Nacidos vivos }\end{array}$ \\
\hline Almoloya de Juárez & 2 & 2,875 & 69.6 \\
\hline Atizapán de Zaragoza & 4 & 7,556 & 52.9 \\
\hline Atlautla & 2 & 520 & 384.6 \\
\hline Chalco & 4 & 5,522 & 72.4 \\
\hline Chicoloapan & 6 & 3,615 & 166.0 \\
\hline Chimalhuacán & 6 & 12,147 & 49.4 \\
\hline Ecatepec de Morelos & 15 & 29,385 & 51.0 \\
\hline Huixquilucan & 3 & 3,755 & 79.9 \\
\hline Ixtapaluca & 5 & 8,323 & 60.1 \\
\hline Ixtlahuaca & 4 & 2,745 & 145.7 \\
\hline Lerma & 2 & 2,120 & 94.3 \\
\hline Naucalpan de Juárez & 8 & 14,009 & 57.1 \\
\hline Nezahualcóyotl & 12 & 18,203 & 65.9 \\
\hline Nicolás Romero & 3 & 6,191 & 48.5 \\
\hline Paz, La & 3 & 4,909 & 61.1 \\
\hline Tecámac & 4 & 5,014 & 79.8 \\
\hline Temascalcingo & 3 & 1,192 & 251.7 \\
\hline Temoaya & 5 & 1,992 & 251.0 \\
\hline Tequixquiac & 2 & 658 & 304.0 \\
\hline Texcoco & 3 & 3,714 & 80.8 \\
\hline Tlalnepantla de Baz & 8 & 9,967 & 80.3 \\
\hline Toluca & 10 & 13,372 & 74.8 \\
\hline Tultitlán & 4 & 8,455 & 47.3 \\
\hline Zumpango & 4 & 2,419 & 165.4 \\
\hline Cuautitlán Izcalli & 4 & 7,090 & 56.4 \\
\hline Valle de Chalco Solidaridad & 3 & 7,968 & 37.7 \\
\hline San José del Rincón & 2 & 1,968 & 101.6 \\
\hline
\end{tabular}

"Municipios con muertes maternas en 2009. Sólo se incluyen los municipios que presentaron dos ó más muertes maternas

Fuente: Secretaria de Salud. Instituto de Salud del Estado de México. Defunciones maternas y Razón de mortalidad materna total por municipio 2009. ${ }^{10}$ 


\section{Objetivo}

Dar a conocer los protocolos y estrategias establecidos en el Hospital General de Ecatepec "Las Américas" en el Estado de México que contribuyen a agilizar la atención en la gestante y coadyuvan en la disminución de la mortalidad materna.

\section{Desarrollo del Contexto}

Para la Organización Panamericana de la Salud (OPS), la mortalidad materna representa un grave problema de salud pública y se ha observado que en el $80 \%$ de los casos las causas son prevenibles, es por eso que en el informe Objetivos de Desarrollo del Milenio se establece el acceso universal a la atención de la salud reproductiva, incluida la planificación familiar, como punto de partida para la salud materna. ${ }^{12}$

Dentro de los factores que se han reconocido como contribuyentes a la ocurrencia de muerte materna están la deficiencia en la identificación oportuna de complicaciones y el retraso para proporcionar atención efectiva. ${ }^{13}$

Por lo anterior se implementó en algunas Unidades de Salud, el Triage Obstétrico el cual es un sistema de clasificación que permite identificar rápidamente la presencia de alguna complicación, catalogar su gravedad y establecer acciones inmediatas. ${ }^{14}$ Para el año 2002, en el Instituto de Salud del Estado de México (ISEM) inicia la política de "cero rechazo" que significa el no rechazar a ninguna mujer embarazada (tenga o no seguro popular), para lo cual se crea el MVI (modulo de valoración individual), atendido por enfermeras perinatales y licenciadas en enfermería y obstetricia. ${ }^{8}$

\section{Triage Obstétrico}

El Triage Obstétrico es un protocolo de atención de primer contacto en emergencias obstétricas, el cual tiene como propósito clasificar la situación de gravedad de las pacientes y precisar la acción necesaria para preservar la vida del binomio o bien la viabilidad de un órgano dentro del lapso terapéutico establecido.

Este sistema se ha adaptado para emplearse en el periodo perinatal (embarazo, parto y puerperio) y en cada contacto de la paciente con el personal de salud. Cuando una paciente es identificada con alguna complicación o emergencia se enlaza y se activa la ruta critica para la vigilancia del embarazo (Código mater).

Se clasifica de acuerdo a su condición clínica de la paciente y de acuerdo a su valoración, se manejan dos distintivos de color, rojo y verde (emergencia y no emergencia respectivamente).

Color Rojo: Atención inmediata, se considera que está comprometida la función vital de la mujer o el feto, puede ser debida a Preeclampsia severa, eclampsia, desprendimiento prematuro de placenta, sépsis puerperal, tromboembolia y embarazadas poli traumatizadas (entre otros).

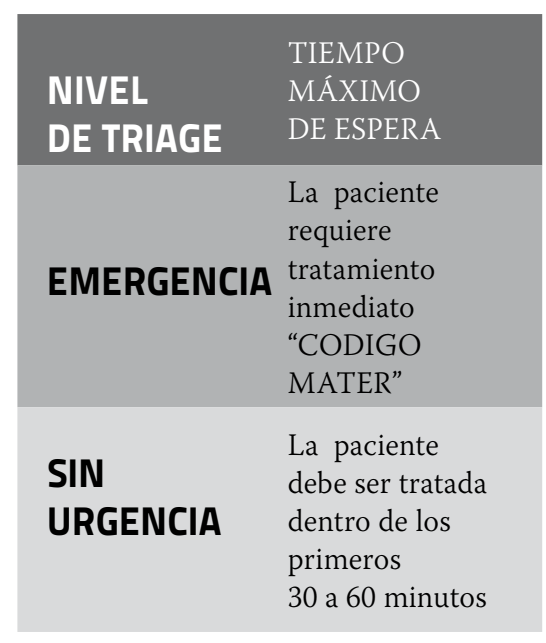

Verde: Pacientes sin antecedentes, en procesos agudos estables, su atención será de 30 a 60 min.

$\mathrm{Al}$ ingresar la paciente al servicio de urgencias Obstétricas, la enfermera perinatal y/o la licenciada en enfermería y obstetricia establecen el primer contacto con la gestante y como primera acción se le toman sus signos vitales, se le realiza un interrogatorio para detectar antecedentes ginecobstétricos de importancia como cefalea, hipertensión, hemorragias, alteración en el estado de alerta, etc. Se toma una muestra de orina para realizar un estudio básico (bililastix), se determina talla y peso. Los datos recabados sirven para llenar las hojas de Triage Obstétrico, con base en esto se determina si el caso es código rojo o verde para pasar a la revisión médica, si se identificara un código rojo la atención médica será inmediata para ser tratado como una urgencia. ${ }^{14}$

\section{MVI (MóduLo DE VALORACIÓN INDIVIDUAL).}

Se requiere que una enfermera perinatal o licenciada en enfermería y obstetricia sean quienes llevan a cabo el Triage Obstétrico, así como los registros correspondientes en la bitácora de ingresos.

Estas profesionales deberán conocer y aplicar perfectamente la escala, realizar una rápida evaluación clínica, registrar los datos expresados por la paciente y dirigir la entrevista, cuando sea necesario, para objetivar al máximo el motivo clínico de consulta y la situación fisiológica de la paciente mediante la monitorización de las constantes vitales (tensión arterial, frecuencia cardiaca, frecuencia respiratoria y temperatura) y documentar dicha valoración. (Tabla 3) 
Tabla 3. Formato actual de la Hoja de Triage Obstétrico

\begin{tabular}{|lll|}
\hline FACTOR & VERDE & ROJO \\
\hline 1. SIGNOS VITALES & & \\
\hline TA & $110-120 / 70-80 \mathrm{mmHg}=$ ó $>$ & $125 / 85 \mathrm{mmHg}=$ ó $<80 / 40 \mathrm{mmHg}$ \\
\hline FC & $60-100$ POR & MÁS DE 100 \\
\hline FR & $18-24$ POR MIN & POR MINUTO \\
\hline TEMP & DE 38 ${ }^{\circ} \mathrm{C}$ & MENOS DE 18 O MÁS DE 24 \\
2. ESTADO DE CONCIENCIA & NORMAL & MÁSUTO MENOS DE $38^{\circ} \mathrm{C}$ \\
\hline 3. HEMORRAGIA TRANSVAGINAL & AUSENTE & ALTERADO \\
\hline 4. CRISIS CONVULSIVAS & AUSENTE & PRESENTE \\
\hline 5. SINTOMAS DE VASOESPASMO & AUSENTE & RESENTE \\
\hline 6. DOLOR EN EPIGASTRIO & AUSENTE & PRESENTE \\
\hline 7. SALIDA DE LIQUIDO AMNIOTICO & AUSENTE & PRESENTE \\
\hline 8. ACTIVIDAD UTERINA & AUSENTE & PRESENTE \\
\hline
\end{tabular}

El responsable del servicio de urgencias, está obligado a tomar las medidas necesarias que aseguren la valoración médica de la usuaria y establecer el tratamiento completo de la urgencia o la estabilización de sus condiciones generales para que pueda ser transferida.

\section{Funciones de la Enfermera Perinatal y/o Licenciada en Enfermería y OBSTETRICIA. ${ }^{15}$}

- Participar en el manejo oportuno y adecuado en la atención de la paciente obstétrica, así como realizar la clasificación de acuerdo al Triage obstétrico establecido.

- Toma y valoración de signos vitales, monitoreo de glucosa en sangre, detección de proteínas en orina, así como realizar los procedimientos necesarios para la correcta clasificación inicial de las pacientes de acuerdo a los signos y síntomas encontrados.

- Realizar una cuidadosa y puntual exploración física, para coadyuvar a determinar tempranamente el diagnóstico médico; así como valorar el ingreso hospitalario, tratamiento ambulatorio o en su caso referencia a otra unidad.
- Conocer y aplicar al 100\% el Triage Obstétrico.

- Realizar el registro del $100 \%$ de las pacientes que son atendidas en el módulo de valoración inmediata.

Respetar la normatividad de ingreso del paciente a urgencias como: a.- Respetar Módulo de Valoración Inmediata para solicitar el servicio. b.- Solicitar al familiar registro en admisión. c.- Solicitar a la paciente $y / 0$ familiar datos personales. d.- Realizar registro en bitácora. e.Toma signos vitales, interrogar so- 
bre datos indicativos de alarma obstétrica. f.- De acuerdo a los datos recabados realizar Triage Obstétrico, colocar distintivo de color correspondiente. Color rojo, notificar de inmediato al médico gineco-obstetra. g.- Brindar apoyo emocional a la paciente, explicar las acciones a seguir y en su caso ingresarla al servicio de Admisión de Toco cirugía.

Todo el personal que labora en el hospital debe conocer el programa de Cero Rechazo en Urgencias.

Una expresión de esta estrategia aplicada en el estado de México se apreció en la disminución de la razón de mortalidad materna del 2005 al 2007 de 76.4 a 46.7 muertes por cada 100 mil nacidos vivos, que representa una disminución de $38.8 \%$ en la RM, sin embargo con base en estas cifras, faltan 27.9 puntos para alcanzar la meta del milenio, lo que equivale a disminuir 3.5 puntos por año. ${ }^{7}$

Se han realizado múltiples acciones en las unidades hospitalarias para coadyuvar en la disminución de la mortalidad materna; en junio del 2007,9 el Hospital General de Ecatepec "Las Américas" le propone a la secretaria de salud la implementación del Código Mater siendo la misma quien autoriza que se lleve a cabo.

El Director, Dr. Efraín Bermúdez Torres ha respaldado la implementación desde su inicio a la actualidad, aunque la aplicación del Código Mater ha presentado modificaciones importantes en cuanto a sus políticas en función de los resultados deseados y los compromisos que se asumen, los siguientes son dos ejemplos de esto. ${ }^{16}$

Del año 2007 al 2009 el Compromiso fue: "Disminuir la Muerte Materna Hospitalaria".
En el año 2011 existieron dos versiones en donde el compromiso fue: "Cero muertes maternas"

\section{CÓDIGO MATER}

Código mater es un sistema de atención de respuesta rápida, concebida como una estrategia hospitalaria para la atención multidisciplinaria de la urgencia obstétrica (hipertensión arterial, sangrado y sépsis).

Lo compone el Equipo multidisciplinario de profesionales de la salud cuya función es optimizar el sistema de atención y los recursos hospitalarios (gineco-obstetra, enfermera, médico intensivistainternista, pediatra, anestesiólogo, inhaloterapista, trabajadora social, laboratorio y ultrasonido), capacitados en urgencias obstétricas, está sustentado en un Proceso $\mathrm{ABC}$.

A Identificar (signos de alarma):

- Hipertensión o hipotensión arterial

- Convulsiones tónico-clónicas

- Alteraciones del sensorio o estado de coma

- Sangrado transvaginal o de otra vía.

- Taquicardia - taquipnea, cianosis, hipertermia $>38.5^{\circ} \mathrm{C}$

- Signos y síntomas de Choque

B Tratar (Seguir guías clínicas establecidas) para el manejo de:

- Preeclampsia-eclampsia

- Sangrado obstétrico

- Sépsis

\section{Trasladar}

- De acuerdo al caso al servicio de Admisión de Tococirugía, Unidad de choque o cuidados intensivos obstétricos.

\section{RUTA CRÍTICA}

1. Ingresa la paciente por sus propios medios o por personal de ambulancia.

2. Es valorada por personal de Triage obstétrico, quien activa código mater mediante alarma sonora o voceo por 5 veces consecutivas.

3. Inicia tratamiento de acuerdo a guías clínicas.

4. Traslado para manejo complementario bien a quirófano o la Unidad de Toco-Cirugía, Unidad de Choque o Cuidados Intensivos Obstétricos.

5. Retroalimentación permanente del sistema, mediante capacitación continua, evaluación crítica de resultados y revisión periódica de la evidencia científica.

\section{FUNCIONES ESPECÍFICAS:}

Médico Gineco-obstetra: valoración integral materno - fetal

- Estado de conciencia

- Estado Cardiopulmonar

- Reflejos osteotendinosos

- Frotis de sangre periférica

- Rastreo ultrasonográfico básico (vitalidad fetal, localización placentaria y calidad del líquido amniótico)

- Exploración pelvi-genital

- Resolución obstétrica quirúrgica.

\section{Licenciada en Enfermería y obstetricia}

Por ser el personal de primer contacto junto con el médico ginecoobstetra es quien vocea o activa el código mater con indicación previa.

Durante la activación de un código mater aproximadamente colaboran cuatro enfermeras; cada una tiene destinadas funciones diferen- 
tes, lo cual da por objetivo: lograr una intervención rápida, oportuna y eficaz a la paciente que presente una urgencia obstétrica. ${ }^{15}$

\section{Funciones:}

- Valoración de signos vitales:

- presión arterial cada 15 minutos

- frecuencia cardiaca

- pulso

- respiración

- temperatura

- Valoración del estado de conciencia

- Instalación de acceso venoso periférico con catéter de calibre grueso.

- Instalación de acceso venoso central.

- Toma de muestras sanguíneas

- Administración de medicamentos; una o dos enfermeras son las encargadas de pasar los medicamentos y otra únicamente de registrarlos en la hoja de enfermería con los datos correctos: vía de administración, dosis, horario y nombre del medicamento.

- Reducción de estímulos que puedan provocar una convulsión.

- Valoración de la presencia de edema.

- Vendaje compresivo de miembros pélvicos.

- Instala sonda vesical para tomar muestra de EGO y cuantificación de líquidos.

- Valorar características de la orina.

- Colocar oxigenación con puntas nasales

- Colocar a la paciente en la posición más óptima para el binomio.
- Valorar Frecuencia Cardiaca Fetal.

- Valorar reflejos osteotendinosos

- Valorar presencia de sangrado transvaginal o de otros sitios.

Médico Intensivista - internista: valoración neurológica, hemodinámica y metabólica a través de:

- Escala de Glasgow

- Cálculo de Gasto cardiaco y resistencias vasculares periféricas.

- Función hepatorrenal (pruebas de función hepática y renal)

- Hidroelectrolítico y ácido-base (electrólitos séricos y gasometría)

- Niveles de glicemia

- Ventilación y capnografía

- Apoyo órgano-funcional y tratamiento multiorgánico.

Médico Pediatra: conocer las condiciones fetales y preparar reanimación neonatal:

- Aplicación de surfactante exógeno

- Apoyo ventilatorio mecánico.

- Reanimación básica y avanzada

- Tratamiento integral del recién nacido.

Médico Anestesiólogo: conocer las condiciones maternas y fetales y adecuar manejo anestésico.

- Intubación oro o naso traqueal.

- Anestesia general inhalatoria.

- Anestesia local regional

- Reanimación hemodinámica con cristaloides, coloides y hemo-derivados.
Trabajadora social: vinculación con los familiares de las pacientes y con otros hospitales o servicios.

- Información sobre estado de salud

- Consentimiento informado para diversos procedimientos

- Trámites administrativos diversos

- Vinculación con centros hospitalarios o servicios en otras unidades médicas

Laboratorio: realizar exámenes solicitados con carácter de urgencia.

- Biometría hemática con cuenta de plaquetas

- Pruebas de coagulación (tiempos de protrombina, trombina y tromboplastina parcial)

- Determinación de glucosa, ácido úrico y creatinina sérica.

- Aminotransferasas y deshidrogenasa láctica

- Gasometría arterial o venosa

- Electrólitos séricos (sodio, potasio y cloro)

- Pruebas de compatibilidad sanguíneas, grupo sanguíneo y factor Rh.

Médico Ultrasonografista: rastreo abdominal y pélvico. Monitorear frecuencia cardiaca, movimientos somáticos, presentación, sitio de inserción placentaria y características morfológicas de la misma, apreciación semi-cuantitativa del líquido amniótico, valoración de reactividad fetal mediante cardiotocografía externa.

\section{Retroalimentación del código mater \\ - Capacitación continua en urgencias obstétricas (desarrollo de destrezas y}


habilidades sobre guías clínicas estatales y federales).

- Discusión dirigida de resultados clínicos hospitalarios.

- Revisión crítica de la evidencia científica actualizada.

\section{Resultados}

El esfuerzo de los diversos gobiernos por disminuir la mortalidad materna no siempre ha sido exitoso. Existen determinantes sociales que inciden en la salud cuya mejoría o solución implica la participación de diversas instancias, el sistema de salud sólo coadyuva para ello, tales como la desigualdad social y la exclusión de la seguridad social. ${ }^{17}$ Sin embargo, las instituciones de salud del Estado de México brindan atención a toda la población en general, aunque se ha establecido como prioridad la salud de los niños y las gestantes.
En el ISEM se ha recopilado evidencia de que la implementación del Triage obstétrico ha logrado reducir tiempos de espera para la consulta obstétrica de 37 minutos a 9.9 minutos; y para las urgencias calificadas de $7.4 \mathrm{mi}$ nutos a 2.2 minutos $^{13}$ (Gráfica 1) colaboran para su realización el personal de primer contacto entre quienes destaca la Licenciada en enfermería o la enfermera especialista perinatal. Por lo cual se puede afirmar que el índice de efectividad del Triage obstétrico es del 100 por ciento.

En el Hospital General de Ecatepec "las Américas" se aprovecharon las evidencias para mejorar progresivamente en beneficio de la salud materna, se realiza así un combate más eficaz a la muerte materna al integrar el código mater, que desde sus inicios ha tenido resultados positivos.
La Gráfica 2 muestra el número de muertes ocurridas en el Hospital General de Ecatepec "Las Américas" desde el año de su apertura (2006) al actual (2012). ${ }^{18}$ El código mater fue implementado a partir del año 2007; durante los dos primeros años que se llevó a la práctica se identificaron fortalezas y debilidades del equipo multidisciplinario que es convocado en la urgencia y de la propia institución.

Las estadísticas muestran los avances obtenidos año tras año en la atención Materna, y evidencian cómo mediante la aplicación de éstas estrategias y protocolos de los que hemos venido hablando, se han logrado cambios significativos en la población; desde el momento en que se logra identificar las diversas formas de manifestación de posibles complicaciones, y a través de establecer un manejo uniforme por parte de todas las especiali-

\section{Gráfica 2. Reducción de tiempo en minutos al realizar una correcta valoración en el Triage Obstétrico}

Minutos

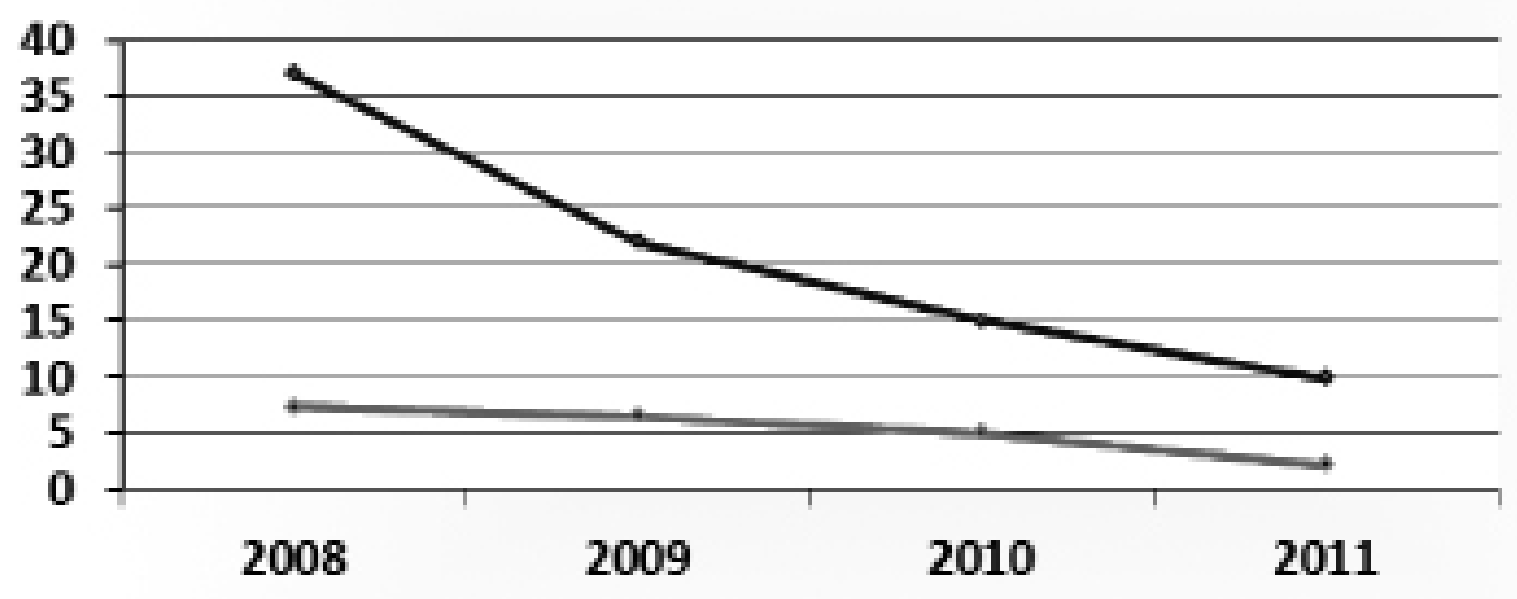

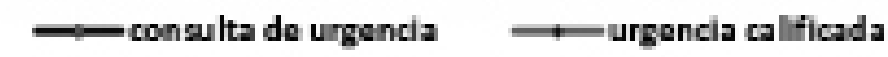




\section{Gráfica 2. Muertes Maternas del año 2007 al 2012 ocurridas en el Hospital General de Ecatepec " Las Américas"*.}

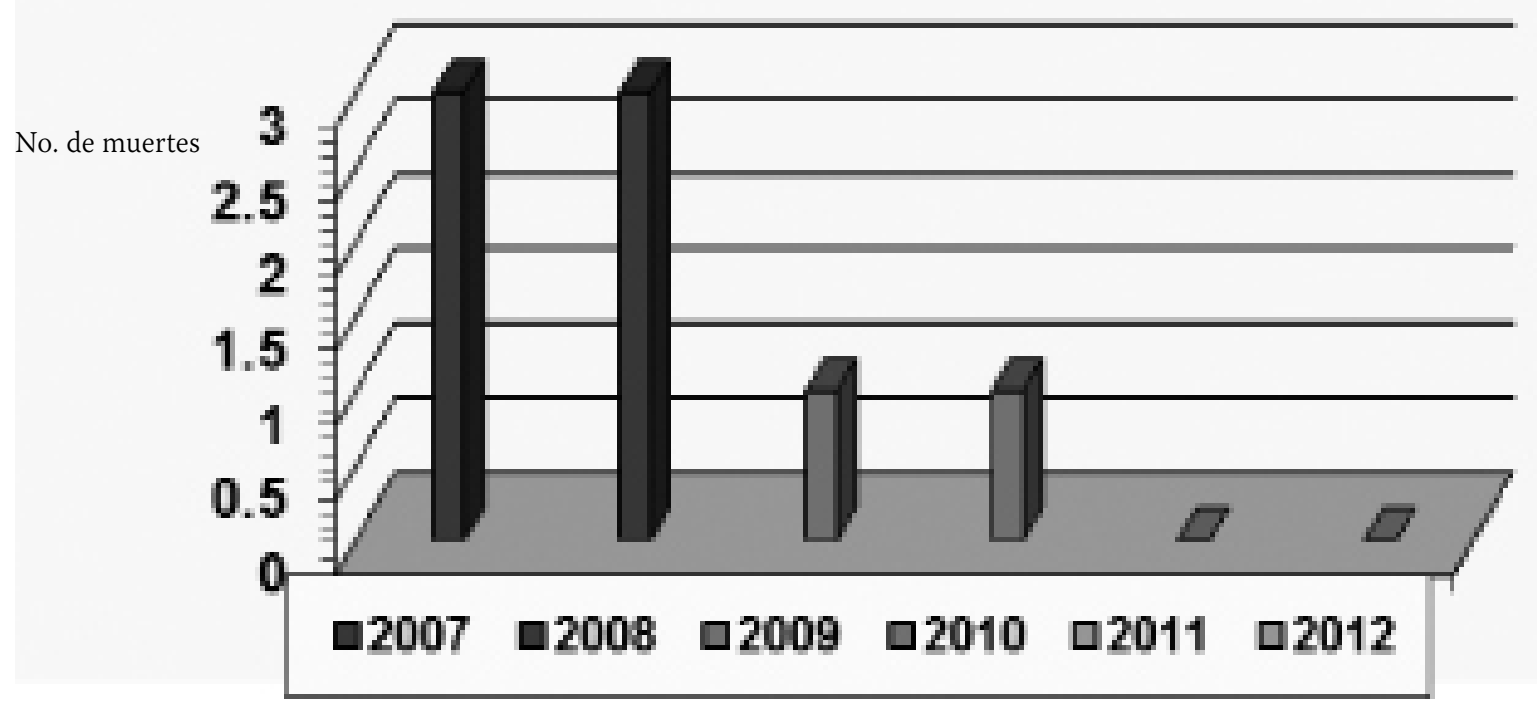

* Departamento de Epidemiología del Hospital General de Ecatepec "Las Américas"

dades competentes, en donde el objetivo principal es seguir los pasos ya establecidos para el mejor pronóstico de la paciente o el binomio, dando un manejo integral correcto y OPORTUNO.

\section{Conclusiones}

La negligencia en la atención proporcionada y el deterioro en los servicios de salud en México han obstaculizado cumplir uno de los Objetivos del Milenio: reducir la tasa de mortalidad materna.

En el presente artículo se muestra como ante el alto índice de morbilidad materna el estado de México ha tomado medidas encaminadas a disminuirla, mediante estrategias que involucren directamente al personal multidisciplinario de salud, a través de la atención oportuna y eficaz.

Estas medidas eran necesarias dado que la entidad mexiquense forma parte de la lista de estados con mayor número de defunciones maternas, los municipios que contribuyen en mayor medida son Nezahualcoyotl, Estado de México y Toluca.

Es importante mencionar que la reducción de la mortalidad materna no requiere costosas inversiones, basta con el acceso a servicios básicos de salud, así como perfeccionar la atención prenatal, y la planificación entre cada embarazo como consultas mensuales durante el embarazo y una unidad de salud con sala de labor y personal calificado durante la atención al parto.

La aplicación del Triage Obstétrico en las instituciones de salud del Estado de México es responsabilidad de la enfermera perinatal o licenciada en enfermería y obstetricia, consiste en una valoración por medio de la cual se identifican fac- tores de riesgo. Cuando se detecta a una mujer con riesgo perinatal o riesgo obstétrico (Preeclampsia, eclampsia, hemorragia obstétrica o sépsis), se activa el sistema de respuesta rápida conocido como Código Mater.

La falla para reconocer de manera temprana el deterioro en la condición clínica de una paciente que la pone en peligro de muerte, es resultado de la poca experiencia del personal de primer contacto con el paciente o la sobrecarga de trabajo que impiden reconocer los signos tempranos de deterioro y responder rápidamente para resolverlos de manera integral, esto fue el fundamento para la creación de los «equipos de respuesta rápida» que tienen la finalidad de reducir las muertes prevenibles, a través de un protocolo de acción que incluye la valoración integral signos de alarma apoyándose en 
la hoja de valoración del Triage obstétrico el cual identifica a las pacientes de urgencias verdaderas que determinan atención inmediata en urgencias obstetricias, durante las 24 horas los 365 días del año.

\section{Referencias Bibliográficas.}

1 Organización Mundial de la Salud. Mortalidad Materna. Nota descriptiva no. 348. Año 2009. [Acceso 19 de Febrero del 2012]. Disponible en: http://bit.ly/OqRi7v

2 Secretaría de Salud. Rendición de Cuentas en Salud 2009. México, SSA, 2010. Disponible en: http:// www.dged.salud.gob.mx

3 Organización de las Naciones Unidas. Objetivos de desarrollo del Milenio. Informe 2010. Nueva York: Naciones Unidas 2010. Disponible en: http://bit.ly/Wq5HVc

4. Díaz-Echeverría DF. Mortalidad Materna. Una tarea Inconclusa. México: Fundar, Centro de Análisis e Investigación, A. C. 2006.

5 Secretaría de Salud. Subsecretaría de prevención y promoción de la salud. Dirección general de epidemiología. México, D.F. Junio, 2011. [Acceso 29 de enero del 2012]. [34 - 35] Disponible en: http://bit.ly/ JHLbNj

6 Gómez Bravo E, Briones-Garduño JC, Morales-Esquivel JG, Rodríguez-Roldán M. Mortalidad Materna en el estado de México. Medicina Crítica y Terapia Intensiva 2003; 17(6):199-203. Disponible en: http://bit.ly/OaBkB5

7 Briones-Garduño JC y cols. Estrategias para reducir la Mortalidad Materna Hospitalaria en el Estado de México. Revista de la Asociación Mexicana de Medicina Crítica [Internet]. Enero a Marzo 2009. [Acceso 17 de febrero del 2012]. 23
(1): [16 - 24]. Disponible en: http:// bit.ly/MBgPb6

8 Secretaria de Salud. Programa Estatal de Salud del Estado de México 2005 - 2011. Política de Cero Rechazo. Toluca, Estado de México. 2005. [acceso 22 de abril del 2012]. [29]. Disponible en: http://bit.ly/ Pf9V2u

9 Salinas-Arnaut A., Villegas-Jiménez M. Guía técnica para El funcionamiento del código mater en las unidades hospitalarias de segundo nivel de atención médica del Instituto de salud del Estado de México. Toluca, Estado de México. Diciembre 2009. [Acceso 26 de febrero del 2012]. Disponible en: http://bit.ly/Krhxg1

10 Secretaria de Salud. Instituto de Salud del Estado de México. Defunciones maternas y Razón de mortalidad materna total por municipio 2009. [Acceso 30 de enero del 2012]. Disponible en: http:// bit.ly/MqkJX0

11 Observatorio de la Mortalidad Materna en México. Numeralia 2010. Diciembre de 2011. Disponible en: http://bit.ly/QMWtjo

12 Organización de las Naciones Unidas. Objetivos del desarrollo del Milenio. Departamento de Información Pública de las Naciones Unidas. New York. Mayo 2005. [acceso 14 de enero del 2012]. [910] Disponible en: http://bit.ly/ M0s3Xy

13 García-Molina FE, Pérez-Santin M, Rodríguez-Tapia A, Sil-Jaimes PA. Triage obstétrico, herramienta en la atención y seguridad de la paciente embarazada y en la reducción de tiempos de espera en el servicio de urgencias de un hospital de Concentración. $9^{\circ}$. Foro Nacional y $6^{\circ}$. Internacional por la Calidad en Salud, 7al 9 de nov
2011, México, D.F. [Acceso 19 de febrero del 2012]. Disponible en: http://bit.ly/QT66Kc

14. Hospital General de Ecatepec "Las Américas". Manual de procedimientos de Ginecología y Obstetricia. Elaboración: 20 de febrero del 2011

15 Grupo de Enfermería Perinatal. Delimitación de funciones de enfermería en el Triage Obstétrico. Hospital General de Ecatepec "Las Américas", 2011.

16 Hospital General de Ecatepec "Las Américas". Manual de procedimientos del área de ginecología y obstetricia. Atención de la paciente obstétrica con Código Mater. 2007.

17 Centro Nacional de Equidad y Género y Salud Reproductiva. Estrategia Integral para acelerar la reducción de la Mortalidad Materna en México. CONEVAL, México [Acceso 29 de enero del 2012]. [3] Disponible en: http://bit.ly/OJGaDe

18 Departamento de Epidemiología. Estadísticas Hospitalarias. Hospital General de Ecatepec "Las Américas". 2007-2012. 


\section{Enfermería Universitaria}

\section{|Lineamientos para Autores}

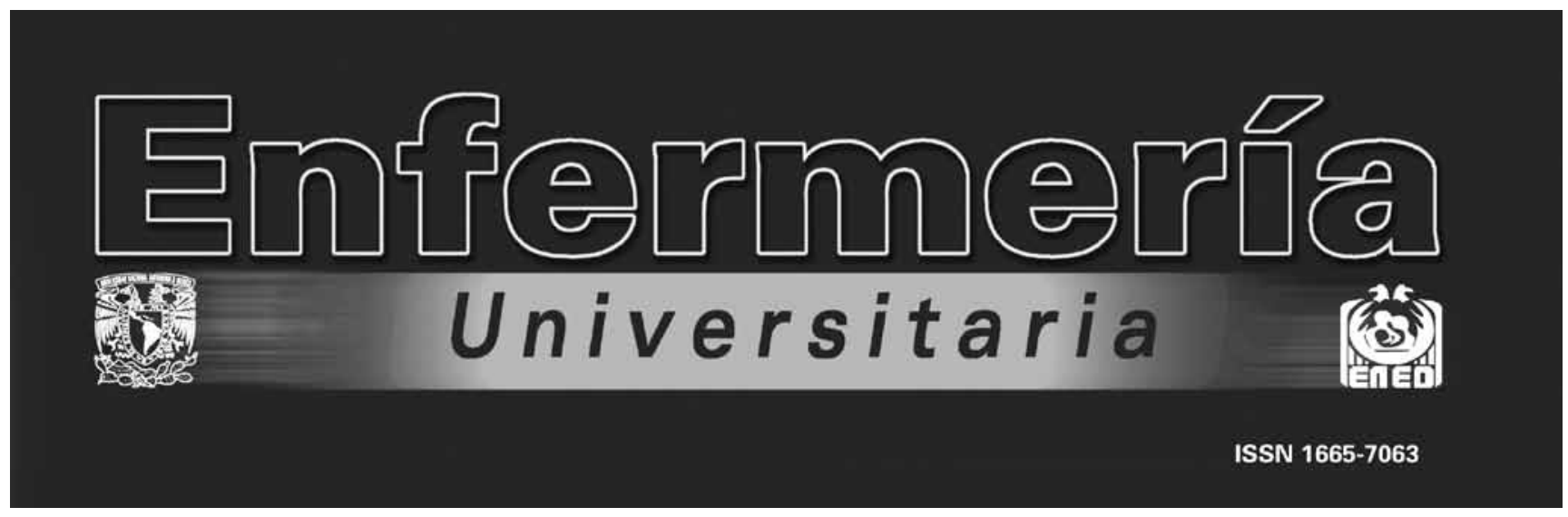

La revista Enfermería Universitaria es una publicación trimestral de la Escuela Nacional de Enfermería y Obstetricia de la UNAM, está indizada en la base de datos CUIDEN de Granada, España, y en el Sistema de Información LATINDEX del Dpto. de Bibliografía Latinoamericana de la Dirección General de Bibliotecas de la UNAM. Tiene como objetivo difundir aportaciones en materia de investigación en salud, educativa y práctica profesional, busca contribuir al fortalecimiento, discusión, e intercambio disciplinar e interdisciplinar con instituciones de salud y académicas, del ámbito nacional e internacional. Pueden participar como autores: profesionales, profesores, y estudiantes de enfermería, y de otras disciplinas de la salud afines.

Políticas de Publicación. La Revista considera para su publicación artículos inéditos, reservándose los derechos exclusivos sobre los mismos. Los conceptos, juicios y opiniones expresados en los artículos serán responsabilidad de las y los autores y no reflejan los criterios, políticas o puntos de vista de la institución y la Revista. La recepción de un trabajo no implica la obligación de publicarlo, ni compromiso con respecto a la fecha de su aparición. Los autores son responsables del contenido de cada uno de los artículos incluyendo cualquier cambio sugerido por el comité revisor.
Proceso de selección. Las contribuciones recibidas pasan por un proceso de selección que dura aproximadamente 4 meses:

1. Dictamen editorial: a la recepción del manuscrito se verifica el cumplimiento de los lineamientos definidos por la revista 
y descritos en las indicaciones a los autores.

2. Dictamen académico: seguidamente se examina el valor académico y científico del documento mediante un sistema de arbitraje por pares (peer review) realizado por expertos en las distintas materias, nacionales y/o internacionales, externos al Comité Editorial. El editor designa dos árbitros y en caso que sus dictámenes discrepen uno del otro se asigna un tercero. Siendo los dictámenes: "Aprobado", "Aprobado con modificaciones" (Se da 1 mes desde su notificación al autor para reenvío con un máximo de 3 reenvíos ) y "No aprobado". En esta fase se protege el anonimato tanto de autores como de revisores.

3. Comité Editorial: con base en los lineamientos de la revista, el valor académico o científico del artículo y la opinión de los árbitros, se define si se publica el artículo o documento presentado.

Las colaboraciones que se reciben deben ser originales e inéditas y responder a alguna de las siguientes secciones:

Editorial. En esta sección un experto opina, analiza y reflexiona sobre un tema novedoso que refleje su experiencia, o bien puede estar dirigido a comentar artículos publicados o a los temas tratados en el número respectivo de la revista.

Artículos de Investigación. Son documentos que tienen como objetivo difundir resultados de investigación original e inédita. Pueden ser investigaciones cuantitativas (se estudia la asociación o relación entre variables cuantificadas) o cualitativas (se centra en el entendimiento de contextos estructurales y situacionales). Pueden ser en formato breve según la concreción de objetivos y resultados.

Artículos de revisión. Tienen como objetivo examinar la investigación publicada sobre un tema en un periodo delimitado y ubicarla en una perspectiva determinada.

Innovación para la práctica. Dentro de esta sección pueden ser incluidos artículos que den a conocer experiencias, reflexiones o propuestas originales, innovadoras y/o relevantes dentro del campo del cuidado, de la gestión (o administración), de la propia investigación o en la educación en enfermería, que hayan marcado o contribuido de manera clara y/o determinante en cambios en esta disciplina.

Cartas al editor. Son cartas sobre temas relevantes para la enfermería o bien relacionadas a los artículos publicados en la revista. Los autores de artículos objeto del comentario, tendrán derecho de réplica.

\section{Preparación del manuscrito}

Entregar impresión original y dos copias en papel bond tamaño carta, letra tipo arial 12, a doble espacio, con paginación inferior lateral derecha empezando con la carátula. Cada parte del escrito en hojas separadas: carátula, resúmenes y palabras clave, texto del artículo, referencias, cuadros, gráficas. Además grabación en $\mathrm{CD}$, con nombres de los archivos y especificación del formato.
El trabajo se acompañará de una carta del autor principal especificando que los manuscritos son inéditos y que no se han enviado simultáneamente a algún otro medio, ni se enviarán antes de conocer la decisión de la Revista. Los autores adjuntarán una declaración firmada indicando que si el documento se acepta para publicación, los derechos de reproducción son propiedad exclusiva de la revista Enfermería Universitaria.

\section{- Carátula}

- Título completo en español y en inglés con extensión máxima de quince palabras que refleje en forma específica y clara el contenido, sin abreviaturas, siglas y sin signos de admiración, interrogación o comillas.

- Título breve en español con extensión máxima de ocho palabras.

- El número máximo de autores para artículos de investigación es seis, y en formato breve tres. El nombre y los apellidos de cada autor se registrarán inmediatamente debajo del título; en notas separadas al pie de la página se anotarán los títulos académicos y cargos de los autores, así como el nombre de la institución de adscripción, la dirección, teléfono, fax y dirección electrónica del autor responsable de la correspondencia y agradecimientos, si es el caso. - Incluir información acerca de cualquier beca o financiamiento recibido.

\section{- Resumen y palabras clave}

Cada manuscrito se acompañará de un resumen en español y en inglés con una extensión no mayor a 250 


\section{Enfermería Universitaria}

palabras, para artículos de investigación señalar: objetivo, metodología, resultados, discusión y conclusiones. Si se trata de otros artículos, el resumen debe incluir: el propósito u objetivos, síntesis del contenido y conclusiones y que no exceda de 200 palabras.

El resumen debe estar seguido por tres a diez palabras clave o descriptores en español (DeCS) y en inglés $(\mathrm{MeSH})$, que facilitan la indexación de los artículos. Para determinarlos se pueden consultar las páginas http://decs.bvs.br/ o http: www.nlm.nih.gov/mesh/MBrowser.html

\section{- Cuerpo del trabajo según sección.}

1. Editorial. Su extensión puede ser entre 2 y 5 cuartillas.

2. Artículos de investigación. Extensión total de 20 cuartillas; para manuscritos en formato breve un máximo de 10 cuartillas. Tanto los trabajos de tipo cuantitativo o cualitativo incluirán los mismos apartados:

- Introducción: Incluir el objetivo y justificación del trabajo con fundamentos teórico-conceptuales. En estudios cualitativos precisar la perspectiva teórica seleccionada.

\section{Metodología:}

Cuantitativos.- En esta sección incluir la descripción de la población de estudio, tamaño de muestra, tipo de muestreo, criterios de selección y pérdida de sujetos. Describir en forma clara y precisa la operacionalización de variables, medición, plan de análisis y métodos estadísticos utilizados, permitiendo así la verificación de los resul- tados. Presentar las pruebas de validez y confiabilidad, de los instrumentos de medición, definir los términos estadísticos, abreviaturas y los símbolos e indicar los programas informáticos utilizados. Cuando se trata de estudios con seres humanos, precisar si los procedimientos empleados han respetado los criterios éticos (Declaración de Helsinki). Adjuntar, en su caso, carta de consentimiento informado y aprobación del comité de ética institucional

Cualitativos.- Brindar información sobre el lugar y contexto del estudio, la selección de los participantes, cómo fueron recolectados los datos, y el tipo de investigación según la posición del investigador, así como los aspectos éticos de la investigación. Descripción del proceso de análisis y de selección de testimonios y de datos contradictorios, sesgos, y participación en el análisis de equipo y participantes.

\section{Resultados:}

Cuantitativos.- Presentarlos con secuencia lógica en el texto. Enfatizar o resumir sólo los hallazgos relevantes y no repetir en el texto los datos de los cuadros, figuras e ilustraciones. Cuidar que el orden y secuencia de éstos sea el correspondiente a la presentación de los mismos.

Cualitativos.- Presentar en forma explícita los resultados, en forma tal que se brinde una imagen significativa del fenómeno bajo estudio.

- Discusión y conclusiones: Se derivan del análisis de los resultados y propósitos del estudio, destacar los aspectos nuevos o relevantes, así como la explicación a los hallazgos encontrados. Comparar los resultados con otros estudios similares, vincular las conclusiones con los objetivos de estudio y cómo contribuye al campo del conocimiento de enfermería. Las afirmaciones deben estar plenamente respaldadas por los datos mostrados, además mencionar las limitaciones del estudio. Para estudios cualitativos es importante que se establezcan las conexiones teóricas pertinentes.

3. Artículos de revisión. Debe participar al menos una persona con experiencia previa en el tema, hacer explícito el universo que cubre esta revisión en cuanto a periodo y bases de datos consultadas, incluye: introducción (con objetivo explícito), desarrollo y conclusiones, en éstas queda explícita la contribución de la revisión en aspectos de la práctica o nuevos elementos de investigación, deben contener mínimo 40 fuentes bibliográficas, pueden incluirse cuadros y figuras,

4. Innovación para la práctica en las siguientes modalidades:

- Información relevante para la práctica. Brindar el contexto de la información, es decir de dónde surge y a quién va dirigida. Información a la que alude (normas, procedimientos, acuerdos, lineamientos, guías clínicas etc.) y fuente(s) de la información.

- Experiencias de la práctica. Introducción, estrategia(s), experiencias, intervención(es), plan de cuidados (si fuera el caso), resultados, conclusiones, referen- 
cias; al menos 5 artículos publicados y 5 textos relativos al tema.

- Revisión crítica de un estudio publicado. Incluirá: Título del comentario, pregunta breve que deberá quedar resuelta al final del resumen, referencia completa del artículo que se comenta, el abstract o resumen del artículo tomado como referencia, comentario realizado por un revisor experto en la materia, explica los motivos por los que se seleccionó el artículo o la importancia de los hallazgos para un área concreta. Citas bibliográficas en las que el revisor base sus comentarios.

- Ensayos. Introducción, desarrollo, conclusiones, referencias; las necesarias para apoyar el manuscrito, preferentemente publicadas en los últimos cinco años.

5. Cartas al editor. Su extensión tendrá un máximo de dos cuartillas.

\section{Cuadros y Figuras.}

$\mathrm{Al}$ diseñar cuadros (tablas) y figuras (dibujos, gráficos y fotografías) tener en cuenta una posible reducción de tamaño. También es importante mencionar que las imágenes o fotografías adquiridas vía internet, no mantienen la resolución requerida para procesarse por lo que debe evitarse su uso. Los encabezados de cuadros y las leyendas de figura deberán entenderse sin hacer referencia al texto. Los pies de figura están señalados con los números arábigos conforme a la secuencia global que les corresponde. El material gráfico constituido por tablas, cuadros y figuras deben presentarse en el orden en que se citan en el trabajo (Tabla 2; Figura 4), en hojas separadas y en el caso de utilizar gráficas estas deberán contar con alta resolución preferentemente archivos JPG.

\section{Referencias Bibliográficas}

Deben seguir estrictamente el modelo Vancouver ${ }^{1}$ utilizado por la mayoría de las revistas biomédicas. Las referencias bibliográficas se numerarán consecutivamente en el orden en que aparecen en el texto y cada una corresponderá a la fuente citada en la lista de referencias bibliográficas al final de la publicación. El número que se le asigna a cada referencia bibliográfica en el texto debe colocarse en números arábigos en superíndice. Una vez que la fuente ha sido citada, el mismo número se utiliza en todas las referencias posteriores. La asignación de los números deben convertirse en identificadores únicos de esa fuente y se reutilizan cada vez que la referencia se cite a lo largo del texto. Para mayor información se recomienda consultar los ejemplos disponibles en Fisterra. com: Estilo Vancouver: Requisitos de uniformidad para manuscritos enviados a revistas biomédicas. http://bit.ly/HTQQcW. A continuación se proporcionan algunos ejemplos para diferentes tipos de documentos.

Libros. Autor/es. Título. Número de Edición. Ciudad de publicación: Editorial; año de publicación. Ejemplo: Burke MM, Walsh MB. Gerontológica: cuidados integrales del adulto mayor. $2^{\text {a }}$ ed. Madrid: Harcourt Brace; 2000.

Capítulo de libro. Autor/es del capítulo. Título del capítulo. En: Editores ó Coordinadores del libro, Título del libro. Número de edi- ción, Ciudad de publicación: Editorial; año de publicación. Páginas inicial y final correspondientes al capítulo. Ejemplo: Jack MQ. Cuidados intensivos en pacientes neurológicos. En: Marino ANET, editores. Cuidados Intensivos Médicos. $3^{a}$ ed México: Interamericana Mc Graw-Hill; 1998. p. 351-366.

Artículos de Revistas. Autor/es. Título del artículo. Abreviatura internacional del título de la revista Año; volumen (número): página inicialfinal del artículo. Ejemplo: Barraza K, Villareal A, Seipp C, Lara P, et al. Prevalencia de asma y otras enfermedades alérgicas en niños escolares. Salud Publica Mex 2005; 43 (3): 433-477.

Algunos autores acostumbran incluir sus dos apellidos, en ese caso citarlos enlazados con un guión ejemplo: Barraza-Colin M, Melgarejo-Ortíz A.

Para las abreviaturas internacionales de los títulos de las revistas se recomienda consultar en: http://1. usa.gov/mHKV2x http://bit.ly/HWbYnP

Documentos Electrónicos. Los documentos tomados de internet citados y referenciados deben cumplir con los siguientes criterios de calidad:

Estar alojados en sitios Web fiables que garanticen estabilidad del contenido con el paso del tiempo; que presenten actualización del sitio; que sean de fácil navegación; que presenten enlaces a otras páginas del sitio y a otros sitios Web con información relacionada y que proporcionen datos de contacto como e-mail, dirección postal, entre otros. 


\section{Enfermería Universitaria}

El documento debe traer fecha de publicación y actualización.

Las citas y las referencias deben presentar consistencia.

El documento debe ser a texto completo y terminado.

La indicación del tipo de documento electrónico se registra entre corchetes. Ejemplo: [Internet]

Para los documentos en línea la fecha de consulta se registra antecedida de la palabra acceso y entre corchetes. Ejemplo: [Acceso 18 de noviembre de 2001]

\section{Artículo de revista de Internet}

Autor/es del artículo. Título del artículo. Título abreviado de la revista [Internet].año mes [fecha de consulta]; volumen (número): [páginas si aparecen].Dirección electrónica. Ejemplo:

Alconero AR, Casaus M, Iglesias R, De la Friera V, Noriega MJ, Fadón A. El corazón de los jóvenes. Enferm Cardiol [Internet]. 2006 [acceso 23 de marzo de 2008]; 13 (38): [13-18]. Disponible en: http:// bit.ly/NYsbYe

\section{Monografía en Internet}

Autor/es o Director/ Coordinador/ Editor. Título [Internet].Edición. Lugar de publicación: Editorial; año [fecha de consulta].Dirección electrónica

\section{Referencia bibliográfica.}

1. Rodríguez- Bonache MJ. ¿Cómo se debe citar en un artículo científico? Rehabilitación (Madr) 2002; 36(2):67-69.

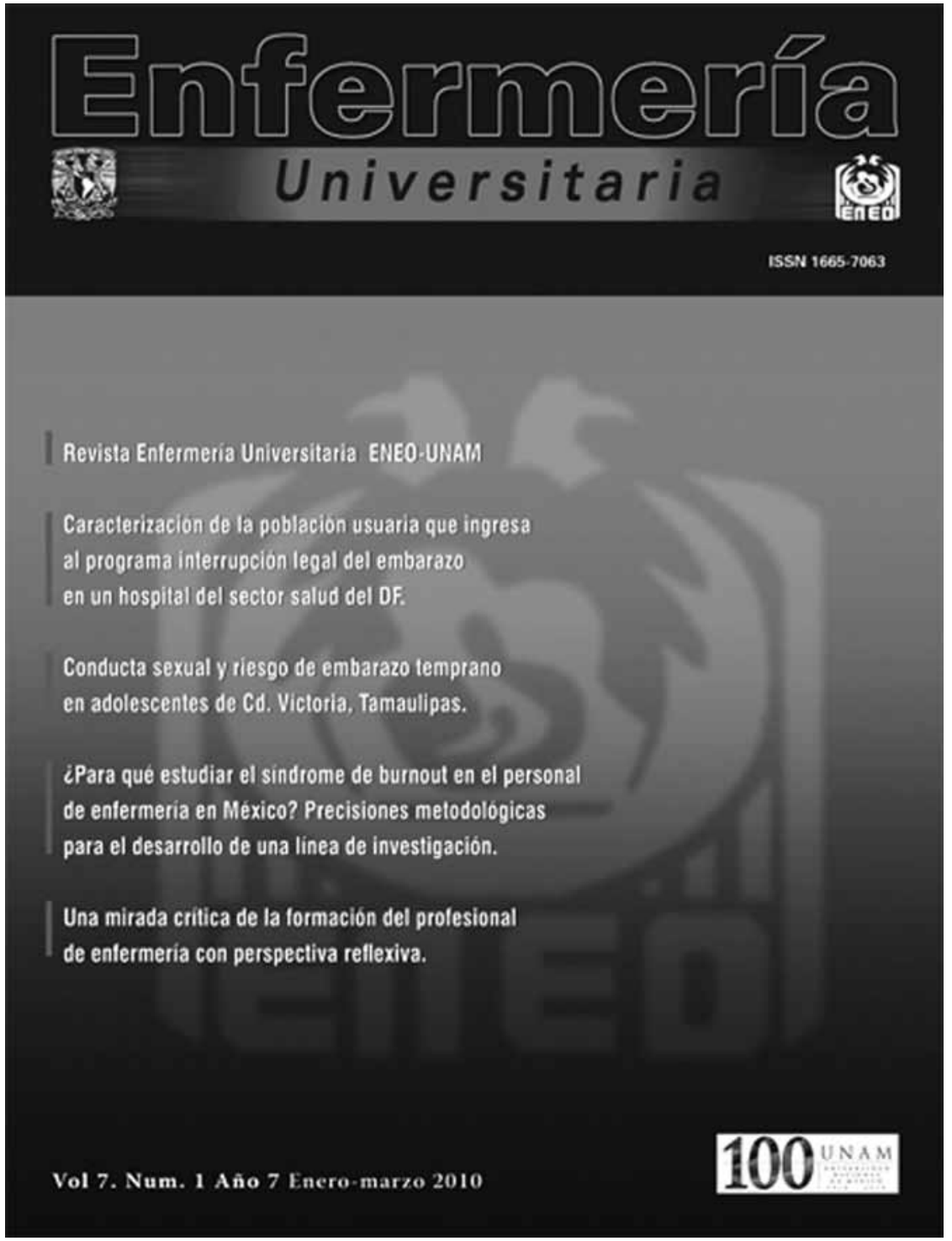

Caracterizacion de la poblacion usuaria que ingresa

al programa interrupcion legal del embarazo

Conducta sexual y riesgo de embarazo temprano

en adolescentes de Cd. Victoria, Tamaulipas.

¿Para que estudiar el sindrome de burnout en el personal

de enfermeria en México? Precisiones metodologicas

para el desarrollo de una linea de investigación.

Una mirada critica de la formación del protesional

de enfermeria con perspectiva reflexiva.

Vol 7. Num. 1 Año 7 Enero-marzo 2010 